\title{
FINITE GROUPS HAVING ONLY ONE IRREDUCIBLE REPRESENTATION OF DEGREE GREATER THAN ONE
}

\author{
GARY SEITZ ${ }^{1}$
}

In this paper all finite groups having exactly one irreducible $K$-representation of degree greater than one are determined, where $K$ is an algebraically closed field of characteristic zero. The quaternion group of order eight and the dihedral group of order eight are nilpotent groups with this property, while the symmetric group on three letters and the alternating group on four letters are solvable although not nilpotent examples. The theorem below will show how typical the above examples really are.

In the following all groups are finite. If $G$ is a group, let $G^{1}$ denote the derived group of $G$ and $Z(G)$ the center of $G$. Let $K$ be an algebraically closed field of characteristic zero.

TheOREM. $A$ group $G$ has exactly one irreducible $K$-representation of degree greater than one if and only if (i) $|G|=2^{k}, k$ is odd, $G^{1}=Z(G)$, and $\left|G^{1}\right|=2$, or (ii) $G$ is isomorphic to the group of all transformations $x \rightarrow a x+b, a \neq 0$, on a field of order $p^{n} \neq 2$.

Proof. By elementary character theory [2] a group $G$ has exactly one irreducible $K$-representation of degree greater than one if and only if $G$ has precisely $\left[G: G^{1}\right]+1$ conjugate classes. Let $g_{0}, \cdots, g_{r}$ be coset representatives of $G^{1}$ in $G$ and suppose $g_{0}=1$. Then $r+2$ $=\left[G: G^{1}\right]+1$. Each coset of $G^{1}$ is a union of conjugate classes, so that $G$ has exactly $\left[G: G^{1}\right]+1$ conjugate classes if and only if each $g_{i} G^{1}$ is a single conjugate class for $i>1$ and $G^{1}$ is the union of the two conjugate classes $\{1\}$ and $G^{1}-\{1\}$.

Suppose that $G$ has exactly one irreducible $K$-representation of degree greater than one. By the above, $G$ acts transitively, by conjugation, on $G^{1}-\{1\}$. Hence $G^{1}$ is elementary abelian and $G^{1}$ is minimal normal in $G$. Moreover, $G$ is solvable since $G^{1}$ is abelian.

If $\left|G^{1}\right|=2$, then $G$ is nilpotent since $G^{1}$ is central in $G$. As each coset of $G^{1}$ other than $G^{1}$ itself is a single conjugate class, $Z(G)=G^{1}$. Thus $G$ is a 2-group, and if $n$ is the degree of an irreducible representation of degree greater than one, then $|G|=\left[G: G^{1}\right]+n^{2}$. This implies that $|G|=2^{k}$ with $k$ odd.

Now let $\left|G^{1}\right|=p^{j}>2$. In this case $Z(G)=1$ because the only

Received by the editors November 8, 1966 and, in revised form, January 10, 1967.

1 Supported by National Science Foundation Grant GP5340. 
conjugate class with a single element is $\{1\}$. Let $C$ be a system normalizer of $G$ [3]. Then $G=C G^{1},[1$, p. 91], and since $G$ is not nilpotent $C \cap G^{1}=1$. The action of $C$ on $G^{1}$, by conjugation, is faithful since $Z(G)=1$ and $C$ is isomorphic to the abelian group $G / G^{1}$. In this representation, $C$ acts irreducibly so that its centralizer is a finite division ring, hence a field. This implies that $C$ is cyclic. The action of $C$ as a permutation group on $G^{1}-\{1\}$ is transitive, and since $C$ is abelian, this action is also regular, $\left[\mathbf{5}\right.$, p. 9], and $|C|=p^{j}-1$. The representation of $G$ on the cosets of $C$ is primitive since $C$ is maximal in $G$. Moreover, this is a faithful representation since $\bigcap_{g} g C^{-1}$ is the hypercenter of $G$ [3] which is trivial. Now using the fact that $|C|=p^{j}-1$ and applying Hilfssatz 2 [4], we then have $G$ isomorphic to the group of all transformations $x \rightarrow a x+b, a \neq 0$, on a field of order $p^{j}$.

For the converse, first suppose

$$
\left|G^{1}\right|=2^{k}, \quad G^{1}=Z(G), \text { and } \quad\left|G^{1}\right|=2 .
$$

Then each $g_{i} G^{1}$ is a single conjugate class for $i>1$ and $g_{0} G^{1}$ splits into two classes, each a singleton. Altogether there are exactly $\left[G: G^{1}\right]+1$ conjugate classes and hence $G$ has precisely one irreducible $K$-representation of degree greater than one.

Now suppose that $G$ is isomorphic to the group of all transformations $x \rightarrow a x+b, a \neq 0$, on a field of order $p^{n} \neq 2$. Then the derived group of $G$ corresponds to the group of all translations. It is easily checked that there are precisely two conjugate classes in $G^{1}$ and any other conjugate class corresponds to all transformations $x \rightarrow a x+b$ for some fixed $0 \neq a \neq 1$. Altogether there are $2+\left(p^{n}-2\right)=p^{n}=\left[G: G^{1}\right]+1$ conjugate classes. This completes the proof of the theorem.

REMARKs. The proof of the theorem showed that if $|G|=2^{k}$, $Z(G)=G^{1}$, and $\left|G^{1}\right|=2$, then $G$ has exactly one irreducible $K$-representation of degree greater than one. Applying the theorem, we then get $k$ to be odd. Hence there is no group $G$ such that $|G|=2^{k}, k$ is even, $Z(G)=G^{1}$, and $\left|G^{1}\right|=2$.

One can obtain examples of the 2-groups appearing in the theorem by taking the direct product of several copies of quaternion or dihedral groups of order eight with amalgamated centers.

I am grateful to Professor Charles Curtis for suggesting the problem of classifying those groups with only one irreducible $K$-representation of degree greater than one. I also wish to thank Professor C. R. B. Wright and the referee for their helpful comments. 


\section{REFERENCES}

1. R. W. Carter, Splitting properties of soluble groups, J. London Math. Soc. 36 (1961), 89-94.

2. C. Curtis and I. Reiner, Representation theory of finite groups and associative algebras, Interscience, New York, 1962.

3. P. Hall, On the system normalizers of a soluble group, Proc. London Math. Soc. (2) 43 (1937), 507-528.

4. B. Huppert, Zweifach transitive, auflösbare Permutationsgruppen, Math Z. 68 (1957), 126-150.

5. H. Wielandt, Finite permutation groups, Academic Press, New York, 1964.

UNIVERSITY OF OREGON 